\title{
THEORY OF CRITICAL SOUND ATTENUATION IN ISING-TYPE MAGNETS
}

\author{
A. PAWLAK \\ Institute of Physics, A. Mickiewicz University \\ Umultowska 85, 61-614 Poznań, Poland
}

(Received May 30, 2000)

\begin{abstract}
The critical behaviour of sound attenuation has been studied in an elastically isotropic Ising system above the critical point on the basis of a complete stochastic model including both spin-energy and lattice-energy modes linearly coupled to the longitudinal sound mode. The effect of spin-lattice relaxation on the ultrasonic attenuation is investigated. The crossover between Kawasaki behaviour and Murata-Iro-Schwabl behaviour is studied as dependent on the values of ultrasonic frequency, reduced temperature, relaxation times, etc. A new high-frequency regime is discussed in the magnetic systems. This new regime corresponds to an adiabatic sound propagation and is very similar to the ones in binary mixture and liquid helium. A new frequency-dependent specific heat being the harmonic average of the bare lattice and critical spin specific heats is introduced. It was shown that such specific heat describes the process of equilibration between spin and lattice subsystems and includes the most important features of critical sound attenuation.
\end{abstract}

PACS numbers: 05.70.Jk, 62.65.+k

\section{Introduction}

Usually a strong anomaly of sound attenuation is measured in Ising like magnetic metals [1, 2]. It is supposed to be described by the theories [3-5] in which the sound wave is assumed to couple to two critical spin fluctuations. The sound attenuation coefficient is proportional then to the imaginary part of the four-spin response function and the critical exponent characterising its divergence is found to be $z \nu+\alpha$, where $z$ is the dynamical critical exponent, $\nu$ is the exponent of the correlation length and $\alpha$ is the exponent of the specific heat.

Contrary to metals, in magnetic insulators we observe a weak anomaly in sound attenuation [1,2], which has been phenomenologically explained by Kawasaki [6] who postulated that in insulators, due to short-range exchange interactions the dominant interaction between the phonon and the spin system is of 
the form of a linear coupling of the longitudinal sound mode to the spin-energy. The latter quantity decays due to the spin-lattice relaxation and thermal diffusion. For the ultrasonic frequencies the spin-lattice (or more correctly spin-energy-lattice-energy) relaxation plays the essential role. Assuming only the specific heat like singularity of the spin-lattice relaxation time and simple separability of contributions coming from both types of coupling, Kawasaki was able to obtain weak divergence of the sound attenuation coefficient proportional to the square of the specific heat for the magnets, in which the first coupling is negligible. However, as shown by the renormalisation-group analysis of the model of coupled spin and energy fields [7], the first assumption is not correct sufficiently close to the critical point where the energy relaxes with the same characteristic critical exponent as the spin fluctuations, $z_{\mathrm{E}}=z$, because the singular part of the energy response function is dominated by the four-spin response function. Also the second assumption concerning the separability fails near $T_{\mathrm{C}}$ as has been recently shown [8].

In this paper we use a complete stochastic model which includes interactions between the scalar order parameter (spin) $S$, the acoustic phonon $Q$, spin-energy $e_{\mathrm{S}}$ and lattice-energy $e_{\mathrm{L}}$ modes in order to find a general expression for the sound attenuation coefficient above the critical temperature. Introduction of the second energy density associated with the lattice (or conduction electrons in some metals) allows us to consider a much richer dynamics than that of the two limiting cases: (i) pure relaxation to a reservoir with infinite thermal conductivity or (ii) pure diffusion of energy. Our model permits also a study of the intermediate dynamics between these limiting cases. The purpose of this paper is to demonstrate that in magnets the critical sound attenuation behaviour is much richer than in other critical systems (as for example liquid helium or critical mixture) owing to the occurrence of two or three different regimes in the same system. It will be shown that the strong sound attenuation anomaly (sometimes called Murata-Iro-Schwabl behaviour) as well as the weak anomaly (Kawasaki behaviour) can be obtained within our stochastic model depending on the relative size of the reduced temperature, frequency and other parameters of the model. The nonuniversal amplitudes for both types of behaviour are modified considerably by the presence of the second energy field in comparison with one-energy-field model. In the high-frequency regime we obtain a new kind of critical behaviour, which we shall call the adiabatic behaviour, analogous to the critical attenuation behaviour in ${ }^{4} \mathrm{He}$ and in binary mixtures. Contrary to the Murata-Iro-Schwabl and Kawasaki behaviour, the amplitude for the adiabatic limit does not change by the inclusion of the field $e_{\mathrm{L}}$.

We shall also show that in some regions of parameters the acoustic self-energy is simply proportional to a frequency-dependent specific-heat $C_{-}$which is the harmonic average of the bare lattice specific heat and the specific heat of an idealised spin system (model A in terminology of Halperin et al. [7]). $C_{-}$has a simple interpretation: it equals to the ratio of the heat transferred from one subsystem to the other, to the induced temperature difference between subsystems. The important point is that such specific heat shows three main types of singularities characterising the sound attenuation coefficient as the critical temperature is approached, for both low and high frequencies. 
The paper is organised as follows. In Sec. 2 we introduce the model and then perform some dynamical decoupling transformations. In Sec. 3 we obtain a general expression for the acoustic self-energy taking into account the reducibility of the latter with respect to energy propagators. In Sec. 4 many different regimes for sound attenuation are discussed as a function of frequency, temperature, and relaxation times. The scaling functions are determined within the one-loop approximation. We then show that for some regions of parameters the ultrasonic attenuation can be written as the imaginary part of $C_{-}(\omega)$. The relevance of this specific heat to the description of nonasymptotic sound attenuation is shown. In Sec. 5 we summarise the results. In Appendix we present Gaussian transformations decoupling the spin and the energy fields.

\section{Model and basic formulas}

The Hamiltonian of the system $[7,8]$ can be written as

$$
\begin{aligned}
H= & \frac{1}{2} \int \mathrm{d}^{d} x\left\{r S^{2}+(\nabla S)^{2}+\frac{u}{2} S^{4}+C_{12}\left(\sum_{\alpha} e_{\alpha \alpha}\right)^{2}+2 C_{44} \sum_{\alpha, \beta} e_{\alpha \beta}^{2}\right. \\
& \left.+2 g \sum_{\alpha} e_{\alpha \alpha} S^{2}+2 f e_{\mathrm{S}} S^{2}+2 w\left(e_{\mathrm{S}}+a e_{\mathrm{L}}\right) \sum_{\alpha} e_{\alpha \alpha}+\frac{e_{\mathrm{S}}^{2}}{C_{\mathrm{S}}}+\frac{e_{\mathrm{L}}^{2}}{C_{\mathrm{L}}}\right\}
\end{aligned}
$$

where $e_{\alpha \beta}(x)$ are components of the strain tensor and the symbols $C_{\alpha \beta}$ stand for the bare elastic constants and $C_{\mathrm{S}}$ and $C_{\mathrm{L}}$ are the spin and the lattice specific heat, respectively. The unitary mass density and $k_{\mathrm{B}} T_{\mathrm{C}}=1$ have been assumed. The first three terms in the total Hamiltonian, describing the static behaviour of the system, make the Ginzburg-Landau functional for the spin variable. The elastic energy is given by the 4th and 5 th term in Eq. (2.1). Here, we have made use of the relation $C_{11}-C_{12}=2 C_{44}$ applicable to the isotropic systems. The last two terms are obtained from the lowest order expansion of the entropy functional with respect to the energy fields. The other terms in the Hamiltonian describe interactions. The constant $g$ denotes the coupling of the (longitudinal) sound mode to two spin fluctuations. The coupling of the sound to the energy fields is characterised by the constants $w$ and $a w$, where the parameter $a$ is the ratio of these two coupling constants, $f$ is the coupling constant between the order parameter and the spin energy generating the divergence of the specific heat.

After introducing the normal mode expansion of the strain tensor [5], the dynamics of the system can be described by the coupled Langevin equations as follows:

$$
\begin{aligned}
& \dot{S}_{\boldsymbol{k}}=-\Gamma \frac{\delta H}{\delta S_{-k}}+\xi_{\boldsymbol{k}}, \\
& \ddot{Q}_{\boldsymbol{k}}=-\frac{\delta H}{\delta Q_{-k}}-\theta k^{2} \dot{Q}_{k}+\eta_{\boldsymbol{k}}, \\
& \dot{e}_{\boldsymbol{k}}^{\mathrm{S}}=-\left(\gamma_{\mathrm{S}}+\lambda_{\mathrm{S}} k^{2}\right) \frac{\delta H}{\delta e_{-\boldsymbol{k}}^{\mathrm{S}}}+\gamma \frac{\delta H}{\delta e_{-k}^{\mathrm{L}}}+\varphi_{\boldsymbol{k}},
\end{aligned}
$$




$$
\dot{e}_{\boldsymbol{k}}^{\mathrm{L}}=-\left(\gamma_{\mathrm{L}}+\lambda_{\mathrm{L}} k^{2}\right) \frac{\delta H}{\delta e_{-\boldsymbol{k}}^{\mathrm{L}}}+\gamma \frac{\delta H}{\delta e_{-\boldsymbol{k}}^{\mathrm{S}}}+\psi_{\boldsymbol{k}},
$$

where $Q_{k}$ is the longitudinal phonon normal coordinate and $\xi_{k}, \eta_{k}, \varphi_{\boldsymbol{k}}$, and $\psi_{\boldsymbol{k}}$ are the Fourier components of Gaussian white noises with variances related to the bare damping terms $\Gamma, \theta k^{2},\left(\gamma_{S}+\lambda_{S} k^{2}\right),\left(\gamma_{L}+\lambda_{L} k^{2}\right)$, and $\gamma$ through the Einstein relations.

The first two of these equations have been commonly used in investigation of critical sound propagation $[4,5,9]$. The other two describe the energy flows between the subsystems. In the absence of the nonlinear terms and noises they transform into the equations describing the decay of the total energy $e=e_{\mathrm{S}}+e_{\mathbf{L}}$ and equalization of temperatures of both subsystems

$$
\begin{aligned}
& \dot{e}=-\frac{\Lambda_{+}}{C_{+}} e-\Lambda_{\Delta e} C_{-} \Delta, \\
& \dot{\Delta}=-\frac{\Lambda_{\Delta e}}{C_{+}} e-\Lambda C_{-} \Delta,
\end{aligned}
$$

where $\Delta=\delta T_{\mathrm{L}}-\delta T_{\mathrm{S}}=e_{\mathrm{L}} / C_{\mathrm{L}}-e_{\mathrm{S}} / C_{\mathrm{S}}$ is the temperature difference between both subsystems, $\Lambda_{+}=\gamma_{\mathrm{S}}+\gamma_{\mathrm{L}}-2 \gamma+\lambda_{+} k^{2}, \Lambda_{\Delta_{e}}=\frac{\gamma_{\mathrm{L}}-\gamma+\lambda_{\mathrm{L}} k^{2}}{C_{\mathrm{L}}}-\frac{\gamma_{\mathrm{S}}-\gamma_{+}+\lambda_{\mathrm{S}} k^{2}}{C_{\mathrm{S}}}, \Lambda=$ $\frac{\gamma_{\mathrm{S}}+\lambda_{\mathrm{S}} k^{2}}{C_{\mathrm{S}}^{2}}+\frac{\gamma_{\mathrm{L}}+\lambda_{\mathrm{L}} k^{2}}{C_{\mathrm{L}}^{2}}+\frac{2 \gamma}{C_{\mathrm{S}} C_{\mathrm{L}}}$, with $\lambda_{+}=\lambda_{\mathrm{S}}+\lambda_{L}, C_{+}=C_{\mathrm{S}}+C_{\mathrm{L}}$ and $C_{-}=$ $\left(\frac{1}{C_{\mathrm{S}}}+\frac{1}{C_{\mathrm{L}}}\right)^{-1}$. If $\gamma_{\mathrm{S}}=\gamma_{\mathrm{L}}=\gamma$, then the total energy is conserved and $\frac{\Lambda_{+}}{C_{+}}=\frac{\lambda_{+}}{C_{+}} k^{2}$ is the rate of the thermal conduction with $\lambda_{\mathrm{S}}$ and $\lambda_{\mathrm{L}}$ being the thermal conductivity for spin and lattice subsystems, respectively. For this case, the temperature difference $\Delta$ decays (in the absence of total energy fluctuations) at the rate $A C_{-} \approx \frac{\gamma}{C_{-}}$. Therefore, $\Lambda C_{-}$can be interpreted as the frequency of the spin-lattice relaxation. It reduces to $\frac{\gamma}{C_{s}}$ for the lattice of infinite specific heat. For non-conserved total energy, as e.g. for $\gamma_{\mathrm{L}}>\gamma_{\mathrm{S}}=\gamma$ (we assume that the spin-energy can change only through interactions with lattice degrees of freedom, i.e. $\gamma_{S}=\gamma$ ),

$$
\begin{aligned}
& \dot{e}=-\frac{\Lambda_{+}}{C_{+}} e-\frac{\Lambda_{+} C_{-}}{C_{\mathrm{L}}} \Delta, \\
& \dot{\Delta}=-\frac{\Lambda_{+}}{C_{+} C_{\mathrm{L}}} e-\Lambda C_{-} \Delta
\end{aligned}
$$

with $\Lambda_{+} \simeq \gamma_{L}-\gamma$. The total energy, in the absence of the temperature difference $\Delta$, decays at the frequency $\frac{\gamma_{L}-\gamma}{C_{+}}$and the temperature difference relaxes (in the absence of total energy fluctuations) at the rate $A C_{-}=\frac{\gamma}{C_{-}}+\frac{\Lambda_{+} C_{\mathrm{S}}}{C_{+} C_{\mathrm{L}}}$ :

In a typical case the additional energy field $e_{\mathrm{L}}$ can be regarded as the sum of energies of all phonon branches except for the longitudinal acoustic phonons with small wave vectors. These phonons are taken explicitly into account in the model, as the quantity of our primary interest in this paper is the phonon response function. For higher frequencies in some metals we can also be interested in the energy flow between the localised spins system and the conduction electrons system. If the lattice degrees of freedom can be treated as an infinite heat bath $\left(C_{\mathrm{L}} \rightarrow \infty\right)$ 
the system may be also described by two energy densities. In that case, the second energy density should be understood as the energy density associated with the spin orientation of conduction electrons. We use the same denotation $e_{\mathrm{L}}$ for this density as the (true) lattice degrees of freedom play only the role of reservoir and do not appear explicitly in the equations of motion. The total energy in the system composed of the localised and conduction electrons is no longer conserved so we have admitted the possibility of nonconserved total energy $e_{\mathrm{L}}+e_{\mathrm{S}}\left(\gamma_{\mathrm{L}} \neq \gamma\right)$ in our general equations of motion.

Instead of using Eq. (2.2a-d), it is convenient to represent the model in terms of the equivalent functional form [10] with a Lagrangian given by

$$
\begin{aligned}
& L=\int_{\omega} \sum_{k}\left\{\Gamma \tilde{S}_{\boldsymbol{k}, \omega} \tilde{S}_{-\boldsymbol{k},-\omega}+\theta k^{2} \tilde{Q}_{\boldsymbol{k}, \omega} \tilde{Q}_{-\boldsymbol{k},-\omega}+\Gamma_{\mathrm{S}}(k) \tilde{e}_{\boldsymbol{k}, \omega}^{\mathrm{S}} \tilde{e}_{-\boldsymbol{k},-\omega}^{\mathrm{S}}\right. \\
& +\Gamma_{\mathrm{L}}(k) \tilde{e}_{\boldsymbol{k}, \omega}^{\mathrm{L}} \tilde{e}_{-\boldsymbol{k},-\omega}^{\mathrm{L}}-2 \gamma \tilde{e}_{\boldsymbol{k}, \omega}^{\mathrm{S}} \tilde{e}_{-\boldsymbol{k},-\omega}^{\mathrm{L}} \\
& -\tilde{Q}_{\boldsymbol{k}, \omega}\left[\left(-\omega^{2}+\mathrm{i} \theta k^{2} \omega\right) Q_{-\boldsymbol{k},-\omega}+\frac{\partial H}{\partial Q_{\boldsymbol{k}, \omega}}\right]-\tilde{S}_{\boldsymbol{k}, \omega}\left(\mathrm{i} \omega S_{-\boldsymbol{k},-\omega}+\Gamma \frac{\partial H}{\partial S_{\boldsymbol{k}, \omega}}\right) \\
& -\tilde{e}_{\boldsymbol{k}, \omega}^{\mathrm{S}}\left[\mathrm{i} \omega e_{-\boldsymbol{k},-\omega}^{\mathrm{S}}+\Gamma_{\mathrm{S}}(k) \frac{\partial H}{\partial e_{\boldsymbol{k}, \omega}^{\mathrm{S}}}-\gamma \frac{\partial H}{\partial e_{\boldsymbol{k}, \omega}^{\mathrm{L}}}\right] \\
& \left.-\tilde{e}_{\boldsymbol{k}, \omega}^{\mathrm{L}}\left[i \omega e_{-\boldsymbol{k},-\omega}^{\mathrm{L}}+\Gamma_{\mathrm{L}}(k) \frac{\partial H}{\partial e_{\boldsymbol{k}, \omega}^{\mathrm{L}}}-\gamma \frac{\partial H}{\partial e_{\boldsymbol{k}, \omega}^{\mathrm{S}}}\right]\right\},
\end{aligned}
$$

where $\tilde{S}, \tilde{Q}, \tilde{e}_{\mathrm{S}}$, and $\tilde{e}_{\mathrm{L}}$ are auxiliary "response" fields and $\Gamma_{i}(k)=\gamma_{i}+\lambda_{i} k^{2}$ for $i=\mathrm{S}, \mathrm{L}$.

First let us decouple fields $\tilde{e}_{\mathrm{S}}, e_{\mathrm{S}}$ from $\tilde{e}_{\mathrm{L}}, e_{\mathrm{L}}$ by the Gaussian transformation

$$
\begin{aligned}
& e_{\boldsymbol{k}, \omega}^{\mathrm{L}} \rightarrow e_{\vec{k}, \omega}^{\mathrm{L}}+\frac{\gamma}{C_{\mathrm{S}} \Gamma_{\mathrm{L}}} D_{\mathrm{L} 0}(k, \omega) e_{\boldsymbol{k}, \omega}^{\mathrm{S}}+A(k, \omega) \tilde{e}_{\boldsymbol{k}, \omega}^{\mathrm{S}}, \\
& \tilde{e}_{\boldsymbol{k}, \omega}^{\mathrm{L}} \rightarrow \tilde{e}_{\boldsymbol{k}, \omega}^{\mathrm{L}}+\frac{\gamma}{C_{\mathrm{L}} \Gamma_{\mathrm{L}}} D_{\mathrm{L} 0}(k,-\omega) \tilde{e}_{\boldsymbol{k}, \omega}^{\mathrm{S}} .
\end{aligned}
$$

Here $D_{\mathrm{L} 0}(k, \omega)=\left[-\mathrm{i} \omega+\Gamma_{\mathrm{L}}(k) C_{\mathrm{L}}^{-1}\right]^{-1}$ is the bare lattice energy propagator and $A(k, \omega)=-2\left[\frac{\gamma}{\Gamma_{\mathrm{L}}} D_{\mathrm{L} 0}(k, \omega)-\frac{\gamma}{C_{\mathrm{L}} \Gamma_{\mathrm{L}}}\left|D_{\mathrm{L} 0}(k, \omega)\right|^{2}\right]$ is a coefficient. For the case of the total energy conserved, this transformation can be also looked at as a subtraction of the slow component associated with thermal conduction mode from $e_{\mathrm{L}}$, so the new variable $e_{\mathrm{L}}$ contains only the fast mode of equalization of temperatures: $e_{\mathrm{L}}^{\text {new }}=$ $C_{\mathrm{L}}\left(\frac{e_{\mathrm{L}}}{C_{\mathrm{L}}}-\frac{e_{\mathrm{S}}}{C_{\mathrm{S}}}\right)$ for $\omega=0$.

As a result of the transformation (2.8), the spin-energy response propagator $D_{\mathrm{S} 0}(k, \omega)=\left[-\mathrm{i} \omega+\Gamma_{\mathrm{S}}(k) C_{\mathrm{S}}^{-1}\right]^{-1}$ is dressed to the form

$\tilde{D}_{\mathrm{SO}}^{-1}(k, \omega)=D_{\mathrm{SO}}^{-1}(k, \omega)-\frac{\gamma^{2} D_{\mathrm{L} 0}(k, \omega)}{\Gamma_{\mathrm{S}} C_{\mathrm{S}} C_{\mathrm{L}}}=C_{\mathrm{S}}{ }^{-1} \Gamma_{\mathrm{S}}\left[1-\mathrm{i} \tilde{\omega}-\frac{\gamma^{2}}{\Gamma_{S} \Gamma_{L}(1-i \bar{\omega})}\right]$,

where $\tilde{\omega}=\omega / \omega_{\mathrm{S}}$ and $\bar{\omega}=\omega / \omega_{\mathrm{L}}$ is the frequency reduced with respect to the bare spin-energy $\omega_{\mathrm{S}}=\Gamma_{\mathrm{S}} / C_{\mathrm{S}}$ and lattice-energy $\omega_{\mathrm{L}}=\Gamma_{\mathrm{L}} / C_{\mathrm{L}}$ relaxation rate, 
respectively. It is easy to see that for $\bar{\omega} \gg 1$, which takes place for $\gamma_{\mathrm{L}} \rightarrow 0$ or $C_{\mathrm{L}} \rightarrow \infty$, the dressed spin-energy propagator reduces to the bare one and the model with two energies reduces to the model without the lattice-energy field, in which only the equation

$$
\dot{e}_{\mathrm{S}}=-\Gamma_{\mathrm{S}}(k) \frac{\delta H}{\delta e_{\mathrm{S}}}+\varphi
$$

matters in our considerations. Depending on the ratio of the bare relaxation rate of the energy diffusion mode $\lambda_{\mathrm{S}} k^{2} / C_{\mathrm{S}}$ to the bare spin-lattice relaxation frequency $\gamma / C_{\mathrm{S}}$, the spin system can be then regarded either as thermally isolated from the lattice $\left(\lambda_{\mathrm{S}} k^{2} / \gamma \gg 1\right.$ and pure energy diffusion takes place) or as freely relaxing to an infinite heat bath $\left(\lambda_{S} k^{2} / \gamma \ll 1\right)$. The reason for introducing the second energy field is now becoming clear. We expect that the model with only one energy density gives a correct description of the dynamics only in the two limiting cases: for pure diffusion and for pure relaxation of the energy field. As we want to be able also to describe the dynamics which is intermediate between these two cases we have included $e_{\mathrm{L}}$ as a separate variable.

\section{Acoustic self-energy}

Having applied (2.8) and other dynamic transformations (A1-A3) decoupling the spin and the energy fields, the acoustic phonon response function $G(k, \omega)=\left\langle\tilde{Q}_{-\boldsymbol{k},-\omega} Q_{k, \omega}\right\rangle$ can be written as follows, to the leading order in the coupling constants $g$ and $w$ :

$$
G(k, \omega)=G_{0}(k, \omega)+G_{0}^{2}(k, \omega) \Sigma^{\mathrm{red}}(k, \omega),
$$

where $G_{0}(k, \omega)=\left(c^{2} k^{2}+\omega^{2}-\mathrm{i} \omega \theta k^{2}\right)^{-1}$ is the free phonon propagator, $c=C_{11}^{1 / 2}$ is the bare sound velocity and

$$
\begin{aligned}
& \Sigma^{\mathrm{red}}(k, \omega)=k^{2}\left[X(k, \omega) \tilde{D}_{\mathrm{LO}}(k, \omega)+Y(k, \omega) \tilde{\tilde{D}}_{\mathrm{SO}}(k, \omega)\right. \\
& \left.\quad+2 g_{1}(k, \omega) g_{2}(k, \omega)\left\langle\Gamma{\widetilde{S^{2}}}_{-\boldsymbol{k},-\omega} S_{\boldsymbol{k}, \omega}^{2}\right\rangle^{L_{\mathrm{etr}}}\right]
\end{aligned}
$$

with ${\widetilde{S^{2}}}_{k, \omega}=(\tilde{S} S)_{\boldsymbol{k}, \omega}$ and $S_{\boldsymbol{k}, \omega}^{2}=\left(S^{2}\right)_{k, \omega}$. The explicit expressions for the coefficients $X(k, \omega), Y(k, \omega), g_{1}(k, \omega)$, and $g_{2}(k, \omega)$ as well as for the dressed propagators $\tilde{D}_{\mathrm{L} 0}(k, \omega)$ and $\tilde{\tilde{D}}_{\mathrm{SO}}(k, \omega)$ are given in Appendix. The crucial point in Eq. (3.2) is that the four-spin response function is calculated with the Lagrangian which depends solely on the spin variables. As the result of coupling of the spin variable to the energy densities and phonons, the effective spin Lagrangian $L_{\text {eff }}$ obtained by transformations $(2.8, \mathrm{~A} 1-\mathrm{A} 3)$ contains phonon- as well as energy-density-mediated four-spin interaction $u(k, \omega) \widetilde{S^{2}}-\boldsymbol{k},-\omega S_{\boldsymbol{k}, \omega}^{2}$ with $u(k, \omega)$ given by (A5). A general expression for the interacting phonon response function is given by

$$
G^{-1}(k, \omega)=G_{0}^{-1}(k, \omega)-\Sigma(k, \omega),
$$

where the phonon self-energy $\Sigma(k, \omega)$ is the irreducible, with respect to phonon lines, part of $\Sigma^{\mathrm{red}}(k, \omega)$. From Eqs. (3.1) and (3.3) the irreducible and reducible parts are related through

$$
\Sigma(k, \omega)=\frac{\Sigma^{\mathrm{red}}(k, \omega)}{1+G_{0}(k, \omega) \Sigma^{\mathrm{red}}(k, \omega)} .
$$


Next we may eliminate the dangerous resonances $[8,9]$ by replacing $G_{0}(k, \omega)$ with $G_{0}(k, 0)$ in $\Sigma(k, \omega)$, i.e. we set the strongly irrelevant parameters, the coefficients at $\omega k^{2}$ and $\omega^{2}$, equal to zero. In this paper we will not discuss the role of the denominator in Eq. (3.4) assuming the elastic couplings $g$ and $w$ to be very small. This assumption gives $\Sigma(k, \omega)=\Sigma^{\text {red }}(k, \omega)$. It is consistent with neglecting the higher order (in $g$ and $w$ ) terms in $\Sigma^{\text {red }}(k, \omega)$. Then also $\tilde{D}_{\mathrm{L} 0}(k, \omega)=D_{\mathrm{L} 0}(k, \omega)$ and $\tilde{\tilde{D}}_{\mathrm{So}}(k, \omega)=\tilde{D}_{\mathrm{SO}}(k, \omega)$. Small values of $g$ and $w$ enable to neglect the macroscopic instability [11], which is believed to take place in compressible spin systems with positive specific-heat exponent near the transition temperature. The weak first-order transition in such systems is a result of a non-analytical character of the coupling constant $u(k, \omega)$ at $k=0$. For small $g$ and $w$ the first-order regime can be probably observed only extremely close to $T_{\mathrm{C}}$.

The perturbation expansion with respect to $u(k, \omega)=u-2 f^{2} N(k, \omega) \Gamma_{\mathrm{S}}$ $\times \tilde{D}_{\mathrm{S} 0}(k, \omega)$ shows that $\left\langle\Gamma \widetilde{S^{2}}-\boldsymbol{k},-\omega S_{\mathbf{k}, \omega}^{2}\right\rangle^{L_{\text {eff }}}$, the four-spin response function, is reducible with respect to (new) spin-energy propagator $\tilde{D}_{\mathrm{S}}$ and so is $\Sigma$. It is easy to show that

$$
\left\langle\Gamma{\widetilde{S^{2}}}_{-\boldsymbol{k},-\omega} S_{\boldsymbol{k}, \omega}^{2}\right\rangle^{L_{\mathrm{eff}}}=\frac{\left\langle\Gamma \widetilde{S^{2}}-k,-\omega S_{\boldsymbol{k}, \omega}^{2}\right\rangle^{\mathrm{irr}}}{1-2 f^{2} \Gamma_{\mathrm{S}} N(k, \omega) \tilde{D}_{\mathrm{S} 0}(k, \omega)\left\langle\Gamma \widetilde{S^{2}}-\boldsymbol{k},-\omega S_{\boldsymbol{k}, \omega}^{2}\right\rangle^{\mathrm{irr}}},
$$

where $\left\langle\Gamma \widetilde{S^{2}}-\boldsymbol{k}_{,-\omega} S_{\boldsymbol{k}, \omega}^{2}\right\rangle^{\mathrm{irr}}$ is an irreducible part of $\left\langle\Gamma \widetilde{S^{2}}-k,-\omega S_{\boldsymbol{k}, \omega}^{2}\right\rangle^{L_{\text {eff }}}$. Equation (3.5) is equivalent to

$$
\left\langle\Gamma{\widetilde{S^{2}}}_{-\boldsymbol{k},-\omega} S_{\boldsymbol{k}, \omega}^{2}\right\rangle^{\mathrm{irr}}=\frac{\left\langle\Gamma \widetilde{S^{2}}-\boldsymbol{k},-\omega S_{\mathbf{k}, \omega}^{2}\right\rangle^{L_{\mathrm{eff}}}}{1+2 f^{2} \Gamma_{\mathrm{S}} N(k, \omega) \tilde{D}_{\mathrm{S} 0}(k, \omega)\left\langle\Gamma{\widetilde{S^{2}}}_{-\boldsymbol{k},-\omega} S_{\boldsymbol{k}, \omega}^{2}\right\rangle^{L_{\mathrm{eff}}}} .
$$

The coefficient preceding $\left\langle\Gamma \widetilde{S^{2}}-k,-\omega S_{\boldsymbol{k}, \omega}^{2}\right\rangle^{L_{\text {eff }}}$ in the denominator of Eq. (3.6) can be written as $2 f^{2} \widehat{D}_{\mathrm{S}}(k, \omega)$ with $\widehat{D}_{\mathrm{S}}(k, \omega)=C_{\mathrm{S}}(1-\mathrm{i} \tilde{\omega} b(k, \omega))^{-1}$. In the $\omega=0$ limit it is equal to $v \equiv 2 f^{2} C_{\mathrm{S}}$. Here $b(k, \omega)=\frac{1-\mathrm{i} \bar{\omega}}{m-\mathrm{i} \bar{\omega}}$ and $m=1-\frac{\gamma^{2}}{\Gamma_{\mathrm{S}}(k) \Gamma_{\mathrm{L}}(k)}$ are coefficients containing the details of the dynamics. As $\left\langle\Gamma \widetilde{S}^{2}-\boldsymbol{k},-\omega S_{\boldsymbol{k}, \omega}^{2}\right\rangle^{\text {irr }}$ is a vertex function, containing no energy resonances, only now we can replace other irrelevant parameters in Eq. (3.6) like the coefficient at $\omega$ in $\tilde{D}_{\mathrm{S} 0}$, in the case of nonconserved systems, by zero. We then obtain

$$
\left\langle\Gamma{\widetilde{S^{2}}}_{-\boldsymbol{k},-\omega} S_{\boldsymbol{k}, \omega}^{2}\right\rangle^{\mathrm{irr}}=\frac{\left\langle\Gamma \widetilde{S^{2}}-\boldsymbol{k},-\omega S_{\boldsymbol{k}, \omega}^{2}\right\rangle^{L_{\mathrm{A}}}}{1+v\left\langle\Gamma \widetilde{S^{2}}-\boldsymbol{k},-\omega S_{\mathbf{k}, \omega}^{2}\right\rangle^{L_{\mathrm{A}}}},
$$

where $L_{\mathrm{A}}$ is the action of the model $\mathrm{A}$ of Halperin et al. [7], with $u_{\mathrm{A}}=u-v$. It is assumed that the coupling constant $v$ does not need to be very small but the positivity of the four-spin coupling $u_{\mathrm{A}}$ must be guaranteed.

In the case of a system with the total energy conserved we cannot proceed in this way as $\tilde{D}_{\text {So }}$ contains nonstatic relevant terms and $u(k, \omega)$ in $L_{\text {eff }}$ contains a pole reflecting conservation of energy and making the action of the model $C$ [7] more appropriate. However, as noted by Halperin et al. [7] if the spin-lattice relaxation is sufficiently fast and the lattice conductivity is so large that the characteristic thermal conduction rate is much faster than the characteristic rate of the order 
parameter, then one does not attain the critical region described by the model $\mathrm{C}$ in the experimentally obtainable range of temperatures. In that case the model $\mathrm{A}$ is more applicable. In the following we assume this situation for our model. A detailed analysis of sound attenuation scaling functions for the model $\mathrm{C}$ will be presented elsewhere.

Now we return to Eq. (3.7) which inserted into Eq. (3.5) yields

$$
\left\langle\Gamma{\widetilde{S^{2}}}_{-\boldsymbol{k},-\omega} S_{\boldsymbol{k}, \omega}^{2}\right\rangle^{L_{\mathrm{eft}}}=\frac{\left\langle\Gamma \widetilde{S^{2}}-\boldsymbol{k},-\omega S_{\boldsymbol{k}, \omega}^{2}\right\rangle^{L_{\mathrm{A}}}}{1+2 f^{2}\left(C_{\mathrm{S}}-\widehat{D}_{\mathrm{S}}\right)\left\langle\Gamma \widetilde{S^{2}}-\boldsymbol{k},-\omega S_{\boldsymbol{k}, \omega}^{2}\right\rangle^{L_{\mathrm{A}}}} .
$$

Next from Eqs. (3.2), (3.4), and (3.8) we obtain

$$
\begin{gathered}
\Sigma(k, \omega)=k^{2}\left[\left\{X(k, \omega) D_{\mathrm{L} 0}(k, \omega)+Y(k, \omega) \tilde{D}_{\mathrm{S} 0}(k, \omega)\right.\right. \\
\left.+\frac{2 g_{1}(k, \omega) g_{2}(k, \omega)\left\langle\Gamma \widetilde{S^{2}}-\boldsymbol{k},-\omega S_{\boldsymbol{k}, \omega}^{2}\right)^{L_{\mathrm{A}}}}{1+2 f^{2}\left(C_{\mathrm{S}}-\widehat{D}_{\mathrm{S}}\right)\left\langle\Gamma \widetilde{S^{2}}-\boldsymbol{k},-\omega S_{\boldsymbol{k}, \omega}^{2}\right\rangle^{L_{\mathrm{A}}}}\right] .
\end{gathered}
$$

It is not difficult to note that $g_{1}(k, \omega)=g_{2}(k, \omega)=\widehat{g}(k, \omega)=g-w f P(k, \omega) \widehat{D}_{\mathrm{S}}(k, \omega)$ with $P(k, \omega)=\frac{m-\mathrm{i} \omega\left(1-a \gamma / \Gamma_{\mathrm{S}}\right)}{m-\mathrm{i} \tilde{\omega}}$. Now let us split $X D_{\mathrm{L} 0}+Y \tilde{D}_{\mathrm{S} 0}$ in Eq. (3.9) into the following terms $\frac{a^{2} w^{2} k^{2} m C_{\mathrm{L}}}{m-\mathrm{i} \bar{\omega}}+w^{2} k^{2} P^{2} \widehat{D}_{\mathrm{S}}(k, \omega)$ and reduce the second term to the common denominator with the third term in Eq. (3.9). This results in the following expression for $\Sigma$ :

$$
\begin{gathered}
\frac{\Sigma(k, \omega)}{k^{2}}=\frac{a^{2} w^{2} C_{\mathrm{L}} m}{m-\mathrm{i} \bar{\omega}} \\
+\frac{\left[2 w^{2} f^{2} P^{2} C_{\mathrm{S}} \widehat{D}_{\mathrm{S}}(k, \omega)-4 w f g P \widehat{D}_{\mathrm{S}}(k, \omega)+2 g^{2}\right]\left\langle\Gamma \widetilde{S^{2}}-\boldsymbol{k},-\omega S_{\boldsymbol{k}, \omega}^{2}\right\rangle^{L_{\mathrm{A}}}+w^{2} P^{2}}{1+2 f^{2}\left(C_{\mathrm{S}}-\widehat{D}_{\mathrm{S}}\right)\left\langle\Gamma \widetilde{S^{2}}-\boldsymbol{k},-\omega S_{\boldsymbol{k}, \omega}^{2}\right\rangle^{L_{\mathrm{A}}}} .
\end{gathered}
$$

Remembering that $C_{\mathrm{S}}-\widehat{D}_{\mathrm{S}}(k, \omega)=-\mathrm{i} \tilde{\omega} b(k, \omega) \widehat{D}_{\mathrm{S}}(k, \omega)$ and ignoring the first noncritical term in Eq. (3.10) the acoustic self-energy can be written in a relatively simple form [12]

$$
\frac{\Sigma(k, \omega)}{k^{2}}=\frac{2\left[g^{2}(k, \omega)-\mathrm{i} \tilde{\omega} b(k, \omega) g^{2}\right]\left\langle\Gamma \widetilde{S^{2}}-k,-\omega S_{k, \omega}^{2}\right\rangle^{L_{\mathrm{A}}}+w^{2} P^{2} C_{\mathrm{S}}}{1-\mathrm{i} \tilde{\omega} b(k, \omega)\left(1+v\left\langle\Gamma \widetilde{S^{2}}-k,-\omega S_{\mathbf{k}, \omega}^{2}\right\rangle^{L_{\mathrm{A}}}\right)}
$$

with an effective frequency-dependent coupling constant $g(k, \omega)=g-w f C_{\mathrm{S}} P(k, \omega)$. The virtue of this formula is that the four-spin response function $\left\langle\Gamma \widetilde{S^{2}}-k,-\omega S_{\mathbf{k}, \omega}^{2}\right\rangle^{L_{\mathrm{A}}}$ is calculated for the phonon and energy free system described by the action $L_{\mathrm{A}}$.

\section{Discussion}

\subsection{General expressions}

The critical contribution to the coefficient of attenuation is determined by the imaginary part of $\Sigma$ and Eq. (3.11) implies 


$$
\alpha(\omega, t)=\left(\frac{\omega}{2 c^{3}}\right) \frac{W_{1}|\Psi|^{2}+W_{2} \operatorname{Im} \Psi+W_{3} \operatorname{Re} \Psi}{|1-\dot{i} \tilde{\omega} b(1+v \Psi)|^{2}},
$$

where the function $\Psi=\left\langle\Gamma \widetilde{S^{2}}-k,-\omega S_{\mathbf{k}, \omega}^{2}\right\rangle^{L_{\mathrm{A}}}$ carries the singularity of the specific-heat type and $W_{1}=2 v \tilde{\omega} \operatorname{Re}\left[\bar{b} g^{2}(k, \omega)\right], W_{2}=2 \operatorname{Re} g^{2}(k, \omega)+2 \tilde{\omega}^{2}|b|^{2} g^{2}+\tilde{\omega} \operatorname{Im}\left[v \bar{b} w^{2} C_{\mathrm{S}} P^{2}+\right.$ $\left.2 b g^{2}-2 \bar{b} g^{2}(k, \omega)\right]$ and $W_{3}=2 \tilde{\omega} \operatorname{Re}\left[\bar{b} g^{2}(k, \omega)-b g^{2}+\bar{b} w^{2} f^{2} C_{\mathrm{S}}{ }^{2} P^{2}\right]+2 \operatorname{Im} g^{2}(k, \omega)$ are nonuniversal coefficients. In the limit $\Gamma_{\mathrm{L}} \rightarrow \infty$ these coefficients take the corresponding values from the model without lattice-energy field [8], i.e. $W_{1}=$ $2 v \tilde{\omega} \tilde{g}^{2}, W_{2}=2\left(\tilde{g}^{2}+\tilde{\omega}^{2} g^{2}\right)$ and $W_{3}=-4 w f C_{\mathrm{S}} \tilde{\omega} \tilde{g}$ with $\tilde{g}=g-w f C_{\mathrm{S}}$.

The four-spin response $\Psi$ function usually is evaluated in the limit $k=0$ since in the ultrasonic experiments the wavelength is much longer than the correlation length $(k \xi \ll 1)$ while the ultrasonic frequency can be comparable to the characteristic frequency of the spin fluctuations. Then the singular part of the response function $\Psi$ obeys the scaling relation

$$
\Psi=t^{-\alpha} \Phi(y)
$$

with $y=\omega t^{-z \nu} / \Gamma$ as the reduced frequency and $t$ proportional to the reduced temperature. The scaling function $\Phi$ is known to the leading order in $\epsilon=4-d$ $[4,5,9]$

$$
\Phi(y)=\Theta^{-\alpha / \nu}\left\{\frac{\nu}{\alpha}+\frac{\mathrm{i}}{y}\left[\mathrm{i}(1 \mathrm{i} y / 2) \arctan (\mathrm{y} / 2)-\frac{1}{2} \ln \left(1+(\mathrm{y} / 2)^{2}\right)\right]\right\} K_{4}
$$

where $\Theta=\left[1+(y / 2)^{2}\right]^{-1 / 4}$ and $K_{4}=\left(8 \pi^{2}\right)^{-1}$.

With the aid of Eq. (4.1) the attenuation coefficient can be written as

$$
\begin{aligned}
\alpha(\omega, t) & =\frac{\omega^{2}}{2 c^{3}}\left[\frac{C_{\mathrm{S}}}{\Gamma_{\mathrm{S}}} \widetilde{W}_{1} t^{-2 \alpha} F_{1}(y ; \omega, t)+\frac{1}{\Gamma} \widetilde{W}_{2} t^{-(\alpha+z \nu)} F_{2}(y ; \omega, t)\right. \\
& \left.+\widetilde{W}_{3} t^{-\alpha} F_{3}(y ; \omega, t)\right],
\end{aligned}
$$

where $\widetilde{W}_{1}=W_{1} K_{4} / \tilde{\omega}, \widetilde{W}_{2}=W_{2} K_{4}, \widetilde{W}_{3}=W_{3} K_{4} / \omega$, and $F_{1}=|\Phi / M|^{2}, F_{2}=$ $\operatorname{Im}(\Phi) y^{-1}|M|^{-2}, F_{3}=\operatorname{Re}(\Phi)|M|^{-2}$ with the denominator of Eq. (3.11) denoted by $M(t, \omega)=1-\mathrm{i} \tilde{\omega} b\left(1+v t^{-\alpha} \Phi\right)$.

\subsection{Regime I}

Equation (4.2) shows many different regimes. First let us consider the regime I defined by the inequality: $m \gg \bar{\omega}$ (equivalent to $\omega \ll \frac{\left(\gamma_{L}-\gamma\right)+\lambda_{+} k^{2}}{C_{L}}$, where $\lambda_{+}=$ $\left.\lambda_{\mathrm{S}}+\lambda_{\mathbf{L}}\right)$. For regime I we have $|b| \rightarrow 1 / m$ and $|P(\omega)| \rightarrow 1$. It seems that the regime I may be adequate for some metals, where $e_{\mathrm{L}}$ should be understood as the spin energy of the conduction electrons and also infinite specific heat associated with phonons can be assumed.

\subsubsection{Low-frequency region}

In the low-frequency region, $\tilde{\omega}|b| \simeq \tilde{\omega} / m \ll 1$, the denominator in Eq. (4.1) does not play any role $(M(t, \omega) \simeq 1)$ and the second term in the numerator with a strong singularity dominates for a sufficiently small reduced temperature $t$

$$
\alpha(\omega, t) \simeq \frac{\tilde{g}^{2}}{c^{3} \Gamma} \omega^{2} t^{-(z \nu+\alpha)} \frac{\operatorname{Im} \Phi(y)}{y} .
$$


This type of singularity in the ultrasonic attenuation, which we shall also call Murata-Iro-Schwabl behaviour, has been so far obtained by neglecting the energy density fields [3,4] and it is believed to take place in magnetic metals $[1,2]$. Note, however, that the coefficient $W_{2}$ in this limit is equal to $2\left(g-w f C_{\mathrm{S}}\right)^{2}$ to be compared with $2 g^{2}$ in the model where the coupling to the energy fields has been neglected [4].

However, if the reduced temperature is not extremely small there may be a competition between the first and the second term in Eq. (4.2). For $t>t_{\text {cross }}$ with $t_{\text {cross }}=\left(\alpha^{2} m \gamma_{\mathrm{S}} / 4 K_{4} \nu^{2} v C_{\mathrm{S}} \Gamma\right)^{1 /(z \nu-\alpha)}$ the weak-singularity term dominates and

$$
\alpha(\omega, t) \simeq \frac{\tilde{g}^{2} v C_{\mathrm{S}}}{c^{3} m \gamma_{\mathrm{S}}} \omega^{2} t^{-2 \alpha}|\Phi(y)|^{2} .
$$

Such behaviour was first obtained by Kawasaki [6]. He postulated phonon-spin-energy coupling in order to explain extremely small sound attenuation exponents observed in magnetic insulators. Note, however, that our scaling function $|\Phi|^{2}$ differs from the classical relaxational function of Kawasaki. What also arises from our analysis, is that such behaviour is generated even if there is no direct coupling of the sound mode to the energy-density fields, and that it cannot be truly asymptotic as both terms (4.3) and (4.4) are proportional (in the $\omega \rightarrow 0$ limit) to the square of the same coupling constant $\tilde{g}$ so even if $g=0$ the strong singularity described by Eq. (4.3) is still present and become dominant for sufficiently small $t$. The dominance of a given type of behaviour does not depend on the relative strength of the coupling constants $g$ and $w$ but rather on the ratio of the bare relaxation times $\gamma_{\mathrm{S}} m / C_{\mathrm{S}} \Gamma$ and also on the ratio of $v$ to its fixed point value $v^{*} \simeq(\alpha / \nu) K_{4}^{-1}$. Slow spin-lattice relaxation (and/or fast decay of spin fluctuations) together with the strong coupling $v$ favour the Kawasaki term.

It should be noted that the amplitudes of the Murata-Iro-Schwabl as well as the Kawasaki term are proportional to the same coupling constant $\tilde{g}^{2}$ only in the limit $\tilde{\omega}=\bar{\omega}=0$. For finite $\tilde{\omega}$ or $\bar{\omega}$ both amplitudes begin to differ from each other and become frequency dependent as can be seen from the expressions for the coefficients $\widetilde{W}_{1}$ and $\widetilde{W}_{2}$. For example $\widetilde{W}_{1}$ is not longer equal to $\widetilde{W}_{2}-2 \tilde{\omega}^{2}|b|^{2} g^{2}$. To be exact this frequency dependence (except $2 \tilde{\omega}^{2}|b|^{2} g^{2}$ term in $\widetilde{W}_{2}$ ) of the coefficients $\widetilde{W}_{i}$ results from the inclusion of the lattice-energy field to the model in which spins, phonons, and spin-energy modes interact. The nonuniversal frequency-dependent coefficients may be useful in making comparisons with experimental results.

\subsubsection{High-frequency region}

As the sound frequency increases, $\tilde{\omega} / m$ approaches unity and the denominator in Eq. (4.1) becomes singular (we assume $v t^{-\alpha} \Phi \gg 1$ ) and $\widetilde{W}_{2}$ (in which now the term $2 g^{2} \tilde{\omega}^{2}|b|^{2}$ dominates) is becoming to be much greater than $\widetilde{W}_{1}$. In that case a new type of sound attenuation behaviour is observed

$$
\alpha(\omega, t) \propto g^{2} \omega^{2} t^{-(z \nu-\alpha)} \frac{\operatorname{Im} \Phi(y)^{-1}}{y},
$$

which can be obtained from the one described by Eq. (4.3) by a simple replacement $\alpha \rightarrow-\alpha, \Phi \rightarrow \Phi^{-1}$ and $\tilde{g} \rightarrow g$. The sound attenuation exponent being 
equal to $z \nu-\alpha$, in this regime, is smaller from the value $z \nu+\alpha$ suitable for the Murata-Iro-Schwabl behaviour, but it is still much larger than $2 \alpha$ characterising the Kawasaki singularity. In this new regime the frequency of the sound mode is much higher than the spin-energy relaxation frequency, so contrary to the low-frequency region described by Eqs. (4.3) and (4.4), now the local temperature of the spin system is not able to follow alternate hot and cold temperature variations produced by the ultrasonic wave. One can say that we have here some kind of adiabatic sound propagation.

As follows from our analysis, on increasing the sound frequency we should observe, in principle, a crossover from Murata-Iro-Schwabl behaviour to the adiabatic behaviour, given by Eq. (4.5), in magnetic systems with a positive specific-heat exponent. The crossover should occur for a frequency of an order of inverse of the spin-lattice relaxation time. The latter quantity plays the key role in the process of Curie-point writing in magneto-optical recording so it has also a technological importance. Unfortunately, there are very few experimental methods able to measure very short spin-lattice relaxation times in magnetic metals. Thus the suggested ultrasonic experiments in which the crossover frequency is measured should provide an interesting new tool to probe the spin-lattice energy transfer rate.

\subsubsection{Comparison of the adiabatic limit with critical ultrasonic attenuation in binary mixtures and liquid ${ }^{4} \mathrm{He}$}

The analytic relation (4.5) resembles the corresponding asymptotic formulas for sound attenuation in binary mixtures [13-15], where the sound attenuation exponent is also equal to $z \nu-\alpha$. For high frequencies Eq. (4.1) can also be transformed into the formula

$$
\alpha(\omega, t) \propto-g^{2} \omega \operatorname{Im}\left(1+v\left\langle\Gamma \widetilde{S^{2}}-k,-\omega S_{k, \omega}^{2}\right)^{L_{\mathrm{A}}}\right)^{-1},
$$

which is identical in form to the expression used by Ferrel and Bhattacharjee [16] for the ultrasonic attenuation near the $\lambda$-transition in liquid helium (although the specific-heat exponent is very close to zero for such systems)

$$
\alpha(\omega, t) \propto-\omega \operatorname{Im} C_{\mathrm{FB}}(t, \omega)^{-1},
$$

where $C_{\mathrm{FB}}(t, \omega)$ is a phenomenological frequency-dependent specific heat. Comparing Eqs.(4.6) and (4.7) we get the correspondence between these equations by interpreting

$$
C_{\mathrm{S}}^{\mathrm{A}}(t, \omega) \equiv C_{\mathrm{S}}\left(1+v\left\langle\Gamma \widetilde{S^{2}}-\boldsymbol{k},-\omega S_{\boldsymbol{k}, \omega}^{2}\right\rangle^{L_{\mathrm{A}}}\right)
$$

as a frequency-dependent specific heat in the Ferrel-Bhattacharjee sense, where as usual we put $k=0$. The function $C_{\mathrm{S}}^{\mathrm{A}}(t, \omega)$ can be relatively simply calculated, as $L_{\mathrm{A}}$ does not contain the details of the dynamics. A very similar identification, giving the statistical meaning to $C_{\mathrm{FB}}(t, \omega)$, was also made by Pankert and Dohm [17] in the case of ${ }^{4} \mathrm{He}$.

The adiabatic formula (4.6) shows a close analogy to the critical sound attenuation in two quite different systems belonging to different universality classes and in the case of ${ }^{4} \mathrm{He}$ even the order parameter dimensionality is different, $n=2$ and $\alpha \simeq 0$. It may suggest that as concerns the adiabatic sound propagation this kind of singularity may be quite common in the critical systems where the order 
parameter is coupled to the energy and sound mode by two different coupling constants. The exception is the gas-liquid system but there, as was noted by Pankert and Dohm [17], the order parameter itself is proportional to the energy and there is only one static coupling between the order parameter and the sound variable.

\subsection{Regime II}

\subsubsection{Low- and high-frequency regions}

Now let us consider a regime which is more appropriate for description of the ordinary lattice consisting mostly of phonons. The new regime, opposite to regime $I$, is defined by the inequality: $m \ll \bar{\omega}\left(\omega \gg \frac{\gamma_{L}-\gamma+\lambda_{+} k^{2}}{C_{L}}\right)$. Now the function $b(\omega) \rightarrow \frac{m+\bar{\omega}^{2}+\mathrm{i} \bar{\omega}(1-m)}{\bar{\omega}^{2}}$. Let us assume further that $m \simeq \frac{\gamma_{L}-\gamma+\lambda+k^{2}}{\Gamma_{\mathrm{L}}(k)} \ll 1$ and $m \ll \bar{\omega}^{2 \dagger}$ then $b(\omega) \simeq 1+\frac{\mathrm{i}}{\bar{\omega}}, P(\omega) \simeq 1-a+\mathrm{i} a \frac{m}{\bar{\omega}}$ and $-\mathrm{i} \tilde{\omega} b(\omega) \simeq \frac{C_{\mathrm{S}}}{C_{\mathrm{L}}}(1-\mathrm{i} \bar{\omega})$. Inserting these expressions into Eq. (3.11) and neglecting the terms of order of $\frac{m}{\bar{\omega}}$, or higher, we get

$$
\begin{aligned}
& \quad \frac{v C_{\mathrm{S}} \Sigma(k, \omega)}{k^{2}}= \\
& \frac{2 \hat{g}^{2} C_{\mathrm{L} 0}(\omega) C_{\mathrm{S}}^{\mathrm{A}}(t, \omega)+2 g^{2} C_{\mathrm{S}} \Delta C_{\mathrm{S}}^{\mathrm{A}}(t, \omega)+\left[w^{2} v(1-a)^{2} C_{\mathrm{S}}-2 \hat{g}^{2}\right] C_{\mathrm{S}} C_{\mathrm{L} 0}(\omega)}{C_{\mathrm{S}}^{\mathrm{A}}(t, \omega)+C_{\mathrm{L} 0}(\omega)}
\end{aligned}
$$

where $C_{\mathrm{L} 0}(\omega)=\frac{C_{\mathrm{L}}}{1-\mathrm{i} \bar{\omega}}$ is the free, frequency-dependent, lattice specific heat, $\widehat{g}=$ $g-w f(1-a) C_{\mathrm{S}}$ and $\Delta C_{\mathrm{S}}^{\mathrm{A}}(t, \omega)=C_{\mathrm{S}}^{\mathrm{A}}(t, \omega)-C_{\mathrm{S}}$ is the fluctuation part of $C_{\mathrm{S}}^{\mathrm{A}}(t, \omega)$.

It is seen from Eq. (4.9) that as long as $\left|C_{\mathrm{L} 0}(\omega)\right| \gg\left|C_{\mathrm{S}}^{\mathrm{A}}(t, \omega)\right|$, i.e. when $\bar{\omega}$ is not too high, the denominator is only slightly singular and we have a kind of competition, with frequency-dependent weights, between two types of low-frequency contributions: the Murata-Iro-Schwabl and Kawasaki term. Note only, the different amplitude, proportional to $\hat{g}^{2}$ in the limit $\tilde{\omega}=0$, instead of $\tilde{g}^{2}$ in Eqs. (4.3) and (4.4). In this low-frequency region the spin and lattice systems stay at equilibrium with each other.

For very high $\bar{\omega}:\left|C_{\mathrm{L} 0}(\omega)\right| \simeq \frac{C_{\mathrm{L}}}{\bar{\omega}} \ll C_{\mathrm{S}}$, which is equivalent to the condition $\tilde{\omega} \gg 1$ the spin and lattice subsystems are no longer in equilibrium and the specific heat $C_{\mathrm{S}}^{\mathrm{A}}(t, \omega)$ begins to outweigh $C_{\mathrm{L} 0}(\omega)$ in the denominator of Eq. (4.9). The acoustic self-energy is dominated by the adiabatic term proportional to

$$
g^{2} \frac{\Delta C_{\mathrm{S}}^{\mathrm{A}}(t, \omega)}{C_{\mathrm{S}}^{\mathrm{A}}(t, \omega)}=\text { const }-g^{2} C_{\mathrm{S}} \frac{1}{C_{\mathrm{S}}^{\mathrm{A}}(t, \omega)} .
$$

\subsubsection{Relevant frequency-dependent specific heats}

Introducing $C_{-}^{\mathrm{A}}(t, \omega) \equiv \frac{C_{\mathrm{LO}}(\omega) C_{\mathrm{S}}^{\mathrm{A}}(t, \omega)}{C_{\mathrm{LO}}(\omega)+C_{\mathrm{S}}^{\mathrm{A}}(t, \omega)}$ and $C_{+}^{\mathrm{A}}(t, \omega) \equiv C_{\mathrm{L} 0}(\omega)+C_{\mathrm{S}}^{\mathrm{A}}(t, \omega)$, Eq. (4.9) can also be written as

$$
\begin{aligned}
& \frac{v C_{\mathrm{S}} \Sigma(k, \omega)}{k^{2}}=2 \hat{g}^{2} C_{-}^{\mathrm{A}}(t, \omega) \\
& \quad+\frac{2 g^{2} C_{\mathrm{S}} \Delta C_{\mathrm{S}}^{\mathrm{A}}(t, \omega)+\left[w^{2} v(1-a)^{2} C_{\mathrm{S}}-2 \hat{g}^{2}\right] C_{\mathrm{S}} C_{\mathrm{L} 0}(\omega)}{C_{+}^{\mathrm{A}}(t, \omega)} .
\end{aligned}
$$

${ }^{\dagger}$ The rare case $m \gg \bar{\omega}^{2}$ was studied in details in Ref. [18]. 
$C_{-}^{\mathrm{A}}(t, \omega)$ is a kind of specific heat which by analogy with the static $(\omega=0)$ case can be given the following interpretation. The energy contribution to the functional $H$, describing the statics, can be rewritten as $\frac{e_{S}^{2}}{C_{\mathrm{S}}}+\frac{e_{\mathrm{L}}^{2}}{C_{\mathrm{L}}}=\frac{e^{2}}{C_{+}}+\frac{e_{\Delta}^{2}}{C_{-}}$, where $1 / C_{-}=$ $1 / C_{\mathrm{S}}+1 / C_{\mathrm{L}}$ and $e_{\Delta}=C_{-}\left(\frac{e_{\mathrm{S}}}{C_{\mathrm{S}}}-\frac{e_{\mathrm{L}}}{C_{\mathrm{L}}}\right)$. If the lattice and the spin systems are at equilibrium with each other, then the local fluctuations of temperature $\delta T_{\mathrm{S}}=\frac{e_{\mathrm{S}}}{C_{\mathrm{S}}}$ and $\delta T_{\mathrm{L}}=\frac{e_{\mathrm{L}}}{C_{\mathrm{L}}}$ are equal, and consequently $e_{\Delta}=0$. Thus $e_{\Delta}$ can be interpreted as excess energy in one system (and deficiency in the other) in comparison with the case when they were at equilibrium with each other $\left(\delta T_{\mathrm{S}}=\delta T_{\mathrm{L}}\right)$, so if we shift the energy $\delta E_{\mathrm{S}}$ from the spin system to the lattice system $\left(\delta E_{\mathrm{L}}=-\delta E_{\mathrm{S}}\right)$ it results in the shifts of temperatures $\delta T_{\mathrm{S}}=\frac{\delta E_{\mathrm{S}}}{C_{\mathrm{S}} V}$ and $\delta T_{\mathrm{L}}=\frac{\delta E_{\mathrm{L}}}{C_{\mathrm{L}} V}=-\frac{\delta E_{\mathrm{S}}}{C_{\mathrm{L}} V}$, where $V$ is the total volume. The total temperature difference $T_{\mathrm{S}}-T_{\mathrm{L}}=\delta T_{\mathrm{S}}-\delta T_{\mathrm{L}}=$ $\frac{\delta E_{S}}{C_{S} V}+\frac{\delta E_{S}}{C_{L} V}=\frac{\delta E_{S}}{C_{-} V}$ is proportional to the energy transported from one system to the other and $C_{-} V=\frac{\delta E_{S}}{T_{S}-T_{L}}$ can be understood as the net heat capacity for the process of differentiation (or equalization) of temperatures between both subsystems, whereas the total specific heat $C_{+}$characterises the thermal properties of the system as the whole (after equilibration).

Coming back to Eq. (4.10) let us note that it can also be rewritten as

$$
\begin{aligned}
& \frac{v C_{\mathrm{S}} \Sigma(k, \omega)}{k^{2}}=2 \hat{g}^{2} \tilde{C}_{-}^{\mathrm{A}}(t, \omega) \\
& +\frac{2\left(g^{2}-\hat{g}^{2}\right) C_{\mathrm{S}} \Delta C_{\mathrm{S}}^{\mathrm{A}}(t, \omega)+w^{2} v(1-a)^{2} C_{\mathrm{S}}^{2} C_{\mathrm{L} 0}(\omega)}{C_{+}^{\mathrm{A}}(t, \omega)},
\end{aligned}
$$

where $\tilde{C}_{-}^{\mathrm{A}}(t, \omega) \equiv \frac{\left(C_{\mathrm{Lo}}(\omega)+C_{\mathrm{S}}\right) \Delta C_{\mathrm{S}}^{\mathrm{A}}(t, \omega)}{C_{\mathrm{L} o}(\omega)+C_{\mathrm{S}}+\Delta C_{\mathrm{S}}^{\mathrm{A}}(t, \omega)}$ may be looked upon again as the net specific heat for the process of equilibration for two subsystems with the only difference that this time the background term $C_{\mathrm{S}}$ has been "moved" from the spin to the lattice subsystem. Now the lattice degrees of freedom plus short-wavelength spin fluctuations constitute the one (noncritical) subsystem and the long-wavelength (critical) spin fluctuations the other. In Eqs. (4.10) and (4.11) the second terms vanish for $g=0$ and $w=0$, respectively. We shall call them the mixing terms because in the case when one of the coupling constants $g$ or $w f(1-a) C_{\mathrm{S}}$ dominates $\hat{g}$, the acoustic self-energy is composed of the dominant term proportional to the corresponding specific heat $\tilde{C}_{-}^{\mathrm{A}}(t, \omega)$ or $C_{-}^{\mathrm{A}}(t, \omega)$ and a small correction term resulting from slightly different ways the constants $g$ and $w$ couple the sound to the spin system. The former one couples the sound mode only to the part of the spin system, the long-wavelength critical spin fluctuations, in contrast to the latter coupling which couples effectively the whole spin system (including the noncritical background) to the sound.

Looking at $\tilde{C}_{-}^{\mathrm{A}}(t, \omega)$ it is easy to see that for $C_{\mathrm{L}} \gg C_{\mathrm{S}}$ it contains both low-frequency singularities: the Murata-Iro-Schwabl and Kawasaki terms, as well as the adiabatic limit. Namely, the first term in

$$
\begin{aligned}
& \operatorname{Im} \tilde{C}_{-}^{\mathrm{A}}(t, \omega)= \\
& \frac{\left|\Delta C_{\mathrm{S}}^{\mathrm{A}}(t, \omega)\right|^{2} \operatorname{Im} C_{\mathrm{L} 0}(\omega)+\left|C_{\mathrm{L} 0}(\omega)+C_{\mathrm{S}}\right|^{2} \operatorname{Im} \Delta C_{\mathrm{S}}^{\mathrm{A}}(t, \omega)}{\left|C_{\mathrm{L} 0}(\omega)+C_{\mathrm{S}}^{\mathrm{A}}(t, \omega)\right|^{2}}
\end{aligned}
$$


can be identified as the Kawasaki term for $\tilde{\omega} \ll 1\left(C_{\mathrm{L} 0}(\omega) \gg C_{\mathrm{S}}^{\mathrm{A}}(t, \omega)\right)$ and the second as the Murata-Iro-Schwabl one. The crossover temperature $t_{\text {cross }}=$ $\left(\frac{\alpha^{2} \gamma}{4 K_{4} \nu^{2} v C_{S} \Gamma}\right)^{1 /(z \nu-\alpha)}$ depends mostly on the ratio of bare relaxation frequencies $\omega_{\mathrm{S}} / \Gamma$. The smaller $\omega_{\mathrm{S}} / \Gamma$ the smaller $t_{\text {cross }}$ and the larger the critical region dominated by the Kawasaki term. In the opposite case $\tilde{\omega} \gg 1, C_{\mathrm{L} 0}(\omega) \ll C_{\mathrm{S}}^{\mathrm{A}}(t, \omega)$ and $\operatorname{Im} \tilde{C}_{-}^{\mathrm{A}}(t, \omega) \simeq C_{\mathrm{S}}^{2} \operatorname{Im} \Delta C_{\mathrm{S}}^{\mathrm{A}}(t, \omega) /\left|C_{\mathrm{S}}^{\mathrm{A}}(t, \omega)\right|^{2}$, i.e. the adiabatic limit is recovered.

The situation described above may be also superimposed by the effects associated with the crossover in the specific heat $C_{\mathrm{S}}^{\mathrm{A}}(t, \omega)$, because the expression for $C_{\mathrm{S}}^{\mathrm{A}}(t, \omega)=C_{\mathrm{S}}\left(1+v t^{-\alpha} \Phi\right)$ may not display the required $t^{-\alpha}$ singularity in the physical region of interest (if $v$ is very small). Then, for example, the measured effective sound attenuation exponent in the Kawasaki region will be much smaller than $2 \alpha$, sometimes close to zero.

\section{Summary}

We have performed a detailed analysis of the critical sound attenuation in magnets. The relaxation of spin-energy to the lattice has been fully taken into account in the prediction of the temperature and frequency dependences of the acoustic self-energy. The important point here is that the acoustic self-energy is reducible with respect to the energy propagators. We were able to express $\Sigma(k, \omega)$ in terms of the four-spin response function $\left\langle\Gamma \widetilde{S^{2}}-\boldsymbol{k},-\omega S_{\boldsymbol{k}, \omega}^{2}\right\rangle^{L_{\mathrm{A}}}$ of the idealised, phonon- and energy-free model $A$. The last quantity can be relatively easy calculated by the renormalisation group method. We have shown that the interpretation of sound attenuation experiments in magnetic systems near their critical points is complicated due to the occurrence of several different regimes, depending on whether or not the energy exchange between the spin and the lattice system is important at the frequency of interest. In the low-frequency region, two kinds of critical behaviour described by Eqs. (4.3) and (4.4) compete with each other. In general their weights are frequency dependent, however, in the limit $\omega \rightarrow 0$ they have a common prefactor proportional to the square of a combination of the coupling constants $g$ and $w$, and their relative ratio is determined mainly by the ratio of the bare relaxation frequencies $\omega_{\mathrm{S}} / \Gamma$. The high-frequency or adiabatic limit is determined completely only by one coupling constant $g$ - describing the interaction of sound mode with two spin fluctuations. The dynamic scaling functions for these three types of singular behaviour are calculated within the one-loop approximation.

Another point of interest is the possibility of expressing the acoustic self-energy by a suitable frequency-dependent specific heat in regime II. We have shown that in the case of one coupling $g$ or $w f C_{\mathrm{S}}(1-a)$ being much stronger than the other, the acoustic self-energy can be very well approximated by the specific heat $C_{-}^{\mathrm{A}}(t, \omega)$ or $\tilde{C}_{-}^{\mathrm{A}}(t, \omega)$, the net specific heats for the process of equilibration between the lattice and spin subsystems or in the latter case between the noncritical background composed of the lattice degrees of freedom plus the noncritical short-wavelength spin fluctuations and the critical subsystem composed of the critical long-wavelength spin fluctuations. The difference in definitions of $C_{-}^{\mathrm{A}}(t, \omega)$ and $\tilde{C}_{-}^{\mathrm{A}}(t, \omega)$ comes from 
slightly different ways the couplings $g$ and $w f C_{\mathrm{S}}(1-a)$ couple the acoustic phonon to the fluctuations of the order parameter.

A similar analysis is also possible for the magnets with the order parameter dimensionality greater than one. For example Eq. (3.11) is still valid there. However, the specific-heat exponent $\alpha$ is negative in $X-Y$ and Heisenberg systems, therefore, the scaling behaviour of the Kawasaki as well as the adiabatic limit will change.

\section{Appendix}

\section{The decoupling transformations}

In this Appendix we present Gaussian transformations decoupling the spin and the spin-energy as well as lattice-energy fields in the Lagrangian. Similarly to Eq. (2.8), which decouples $\tilde{e}_{S}$, es from $\tilde{e}_{\mathrm{L}}$, e e decouple the sound mode from the spin and the energy fluctuations by the shift of variables

$$
\begin{aligned}
Q_{\boldsymbol{k}, \omega} & \rightarrow Q_{\boldsymbol{k}, \omega}-g k G_{0}(k, \omega) S_{\boldsymbol{k}, \omega}^{2}-w k J(k, \omega) G_{0}(k, \omega) e_{\boldsymbol{k}, \omega}^{\mathrm{S}}-a w k G_{0}(k, \omega) e_{\boldsymbol{k}, \omega}^{\mathrm{L}} \\
& +B(k, \omega){\widetilde{S^{2}}}_{\boldsymbol{k}, \omega}+E(k, \omega) \tilde{e}_{\boldsymbol{k}, \omega}^{\mathrm{S}}+F(k, \omega) \tilde{e}_{\boldsymbol{k}, \omega}^{\mathrm{L}}, \\
\tilde{Q}_{\boldsymbol{k}, \omega} & \rightarrow \tilde{Q}_{\boldsymbol{k}, \omega}-2 g k G_{0}(k,-\omega) \Gamma{\widetilde{S^{2}}}_{\boldsymbol{k}, \omega}-w k K(k,-\omega) \Gamma_{\mathrm{S}} G_{0}(k,-\omega) \tilde{e}_{\boldsymbol{k}, \omega}^{\mathrm{S}} \\
& -w k\left(a \Gamma_{\mathrm{L}}-\gamma\right) G_{0}(k,-\omega) \tilde{e}_{\boldsymbol{k}, \omega}^{\mathrm{L}} .
\end{aligned}
$$

Here the abbreviations $B(k, \omega)=-4 \Gamma g k\left|G_{0}\right|^{2} \theta k^{2}, J(k, \omega)=1+\frac{a \gamma D_{\mathrm{L} 0}(k, \omega)}{C_{\mathrm{S}}}$, $F(k, \omega)=-2 w k\left(a \Gamma_{\mathrm{L}}-\gamma\right)\left|G_{0}\right|^{2} \theta k^{2}, E(k, \omega)=-2 w k K(k,-\omega) \Gamma_{\mathrm{S}}\left|G_{0}\right|^{2} \theta k^{2}-$ $\operatorname{awk} A(k, \omega) G_{0}(k, \omega), K(k, \omega)=N(k, \omega)-\frac{a \gamma}{\Gamma_{\mathrm{S}}}\left(1-\frac{D_{\mathrm{L} 0}(k, \omega) \Gamma_{\mathrm{L}}}{C_{\mathrm{L}}}\right), N(k, \omega)=1-$ $\frac{\gamma^{2} D_{\mathrm{LO}}(k, \omega)}{C_{\mathrm{L}} \Gamma_{\mathrm{S}}}$ have been introduced. As an undesirable by-product of this transformation we get the spin-energy and lattice-energy fields coupled again in spite of transformation (2.8). However, this coupling can be removed by the following transformation:

$$
\begin{aligned}
e_{\boldsymbol{k}, \omega}^{\mathrm{L}} & \rightarrow e_{\boldsymbol{k}, \omega}^{\mathrm{L}}+R(k, \omega) S_{\boldsymbol{k}, \omega}^{2}+T(k, \omega) e_{\boldsymbol{k}, \omega}^{\mathrm{S}}+U(k, \omega) \widetilde{S^{2}}{ }_{k, \omega}+V(k, \omega) \tilde{e}_{\boldsymbol{k}, \omega}^{\mathrm{S}}, \\
\tilde{e}_{\boldsymbol{k}, \omega}^{\mathrm{L}} & \rightarrow \tilde{e}_{\boldsymbol{k}, \omega}^{\mathrm{L}}+\tilde{D}_{\mathrm{L} 0}(k,-\omega)\left[2 a w g k^{2} G_{0}(k,-\omega) \Gamma{\widetilde{S^{2}}}_{\boldsymbol{k}, \omega}\right. \\
& \left.+a w^{2} k^{2} K(k,-\omega) G_{0}(k,-\omega) \Gamma_{\mathrm{S}} \tilde{e}_{\boldsymbol{k}, \omega}^{\mathrm{S}}\right],
\end{aligned}
$$

which decouples $\tilde{e}_{\mathrm{L}}, e_{\mathrm{L}}$ from the spin and the spin-energy fluctuations. Here, the abbreviations are

$$
\begin{aligned}
& R(k, \omega)=\left[\gamma f+\left(a \Gamma_{\mathrm{L}}-\gamma\right) w g k^{2} G_{0}(k, \omega)\right] \tilde{D}_{\mathrm{L} 0}(k, \omega), \\
& T(k, \omega)=w^{2} k^{2} J(k, \omega)\left(a \Gamma_{\mathrm{L}}-\gamma\right) G_{0}(k, \omega) \tilde{D}_{\mathrm{L} 0}(k, \omega), \\
& U(k, \omega)=4 \Gamma w g k^{2}\left(a \Gamma_{\mathrm{L}}-\gamma\right)\left|G_{0}\right|^{2} \theta k^{2} \tilde{D}_{\mathrm{L} 0}(k, \omega) \\
& \quad+4 a g w k^{2} \Gamma \Gamma_{\mathrm{L}} G_{0}(k,-\omega)\left|\tilde{D}_{\mathrm{L} 0}\right|^{2} \\
& V(k, \omega)=w k\left\{2 a w k K(k,-\omega)\left[1+\left(a \Gamma_{\mathrm{L}}-\gamma\right) w^{2} k^{2}\left|G_{0}\right|^{2} \theta k^{2}\right]\right. \\
& \left.\quad \times G_{0}(k,-\omega)\left|\tilde{D}_{\mathrm{L} 0}\right|^{2} \Gamma_{\mathrm{S}} \Gamma_{\mathrm{L}}-\left(a \Gamma_{\mathrm{L}}-\gamma\right) E(k,-\omega) \tilde{D}_{\mathrm{L} 0}(k, \omega)\right\}
\end{aligned}
$$




$$
\begin{gathered}
W(k, \omega)=2 w g k^{2}\left[a G_{0}(k,-\omega) A(k, \omega)+2 K(k, \omega) \Gamma_{\mathrm{S}}\left|G_{0}\right|^{2} \theta k^{2}\right] \Gamma \tilde{\tilde{D}}_{\mathrm{S} 0}(k, \omega) \\
-4\left[f-w g k^{2} J(k,-\omega) G_{0}(k,-\omega)\right]\left(1-\frac{\gamma^{2} \Gamma_{\mathrm{L}} \Gamma\left|D_{\mathrm{L} 0}\right|^{2}}{\Gamma_{\mathrm{S}} C_{\mathrm{L}}^{2}}\right) \Gamma \Gamma_{\mathrm{S}}\left|\tilde{\tilde{D}}_{\mathrm{S} 0}\right|^{2}
\end{gathered}
$$

Finally, we apply the transformation

$$
\begin{aligned}
e_{\boldsymbol{k}, \omega}^{\mathrm{S}} & \rightarrow e_{\boldsymbol{k}, \omega}^{\mathrm{S}}+\tilde{\tilde{D}}_{\mathrm{S} 0}(k, \omega) \Gamma_{\mathrm{S}}\left[w g k^{2} K(k, \omega) G_{0}(k, \omega)-f N(k, \omega)\right] S_{\boldsymbol{k}, \omega}^{2} \\
& +W(k, \omega){\widetilde{S^{2}}}_{\boldsymbol{k}, \omega} \\
\tilde{e}_{\boldsymbol{k}, \omega}^{\mathrm{S}} & \rightarrow \tilde{e}_{\boldsymbol{k}, \omega}^{\mathrm{S}}-2 \tilde{\tilde{D}}_{\mathrm{S} 0}(k,-\omega)\left[f-w g k^{2} J(k,-\omega) G_{0}(k,-\omega)\right] \Gamma \widetilde{S^{2}}{ }_{k, \omega}
\end{aligned}
$$

decoupling $\tilde{e}_{\mathrm{S}}$, e from the spin fluctuations, where $\tilde{D}_{\mathrm{L} 0}^{-1}(k, \omega)=D_{\mathrm{L} 0}^{-1}(k, \omega)-$ $\left(a \Gamma_{\mathbf{L}}-\gamma\right) a w^{2} k^{2} G_{0}(k, \omega)$ and $\tilde{\tilde{D}}_{\mathrm{S} 0}^{-1}(k, \omega)=\tilde{D}_{\mathrm{S} 0}^{-1}(k, \omega)-w^{2} g^{2} k^{2} J(k, \omega) K(k, \omega)$ $\times G_{0}(k, \omega)$, are "dressed" by transformations (2.8) and (A1) energy-density propagators.

As a result of transformations (2.8) and (A1-A3), the Lagrangian can be written as a sum of independent parts

$$
L=L_{\mathrm{eff}}(S, \tilde{S})+L_{\mathrm{ph}}(Q, \tilde{Q})+L_{\mathrm{se}}\left(e_{\mathrm{S}}, \tilde{e}_{\mathrm{S}}\right)+L_{\mathrm{le}}\left(e_{\mathrm{L}}, \tilde{e}_{\mathrm{L}}\right)
$$

describing the spin and the new phonon, spin-energy and lattice-energy variables, respectively. $L_{\mathrm{ph}}(Q, \tilde{Q}), L_{\mathrm{se}}\left(e_{\mathrm{S}}, \tilde{e}_{\mathrm{S}}\right)$ and $L_{\mathrm{le}}\left(e_{\mathrm{L}}, \tilde{e}_{\mathrm{L}}\right)$ have harmonic forms and the effective spin Lagrangian $L_{\text {eff }}(S, \tilde{S})$ contains phonon- as well as energy-density-mediated four-spin non-local interactions the most important of which is $u(k, \omega) \widetilde{S^{2}}-\boldsymbol{k},-\omega S_{\boldsymbol{k}, \omega}^{2}$ with the renormalised coupling constant

$$
\begin{aligned}
& u(k, \omega)=u-2 g^{2} k^{2} G_{0}(k, \omega)-2 a w g k^{2} \tilde{f} \Gamma_{\mathrm{L}} G_{0}(k, \omega) \tilde{D}_{\mathrm{L} 0}(k, \omega) \\
& -2 \hat{f}_{1} \hat{f}_{2} \Gamma_{\mathrm{S}} \tilde{\tilde{D}}_{\mathrm{S} 0}(k, \omega),
\end{aligned}
$$

where $\tilde{f}=\gamma f+\left(a \Gamma_{\mathrm{L}}-\gamma\right) w g k^{2} G_{0}(k, \omega) ; \hat{f}_{1}=f N(k, \omega)-w g k^{2} K(k, \omega) G_{0}(k, \omega)$ and $\hat{f}_{2}=f-w g k^{2} J(k, \omega) G_{0}(k, \omega)$.

After the transformations of variables, the acoustic phonon response function can be written in the form given by Eqs. (3.1) and (3.2) with the coefficients $X(k, \omega)=a\left(a \Gamma_{\mathrm{L}}-\gamma\right) w^{2}, Y(k, \omega)=w^{2} \Gamma_{\mathrm{S}} K(k, \omega) J(k, \omega)$, $g_{1}(k, \omega)=g-w \hat{f}_{1} \Gamma_{\mathrm{S}} K(k, \omega) \tilde{\tilde{D}}_{\mathrm{S} 0}(k, \omega)+a w \tilde{f} \Gamma_{\mathrm{L}} \tilde{D}_{\mathrm{L} 0}(k, \omega)$ and $g_{2}(k, \omega)=$ $g-w \hat{f}_{2} J(k, \omega) \Gamma_{\mathrm{S}} \tilde{\tilde{D}}_{\mathrm{S} 0}(k, \omega)$.

\section{References}

[1] B. Lüthi, in: Dynamical Properties of Solids, Eds. G.K. Horton, A.A. Marudin, Vol. 3, North-Holland, Amsterdam 1980, p. 245.

[2] K. Kawasaki, in: Phase Transitions and Critical Phenomena, Eds. C. Domb, M.S. Green, Vol. 5a, Academic Press, New York 1976, p. 165.

[3] K.K. Murata, Phys. Rev. B 13, 4015 (1976).

[4] H. Iro, F. Schwabl, Solid State Commun. 46, 205 (1983).

[5] A. Pawlak, J. Phys., Condens. Matter 1, 7989 (1989).

[6] K. Kawasaki, Phys. Lett. A 29, 406 (1968). 
[7] B.I. Halperin, P.C. Hohenberg, S. Ma, Phys. Rev. B 10, 139 (1974).

[8] A. Pawlak, Eur. Phys. J. B 4, 179 (1998).

[9] R. Dengler, F. Schwabl, Z. Phys. B, Condens. Matter 69, 327 (1987).

[10] H.K. Janssen, Z. Phys. B, Condens. Matter 23, 377 (1976).

[11] D.J. Bergman, B.I. Halperin, Phys. Rev. B 13, 2145 (1976).

[12] A. Pawlak, Acta Phys. Pol. A 97, 909 (2000).

[13] D.M. Kroll, J.M. Ruhland, Phys. Rev. A 23, 371 (1981).

[14] R.A. Ferrell, J.K. Bhattacharjee, Phys. Rev. A 31, 1788 (1985).

[15] R. Folk, G. Moser, Phys. Rev. E 58, 6246 (1998).

[16] R.A. Ferrell, J.K. Bhattacharjee, Phys. Rev. Lett. 44, 403 (1980); Phys. Rev. B 23, 2434 (1981).

[17] J. Pankert, V. Dohm, Phys. Rev. B 40, 10842, 10856 (1989).

[18] A. Pawlak, M. Błaszak, Acta Phys. Pol. A 97, 905 (2000). 